\title{
Facing the complexity of Alzheimer's disease
}

\author{
Patrick Dallemagne ${ }^{1}$ \& Christophe Rochais*,1 (iD \\ ${ }^{1}$ Normandie Univ, UNICAEN, CERMN, Caen, France \\ *Author for correspondence: christophe.rochais@unicaen.fr
"Considering the multifactorial origin of $A D$, the development of selective agents might not be sufficiently active to reverse the cognitive deficit."

First draft submitted: 4 November 2019; Accepted for publication: 13 November 2019; Published online: 21 November 2019

Keywords: Alzheimer's disease • animal models • clinical trials • polypharmacology

\begin{abstract}
Alzheimer's disease (AD) currently represents the most common form of dementia in elderly people, and with the increase in life expectancy the number of patients suffering from $\mathrm{AD}$ is expected to increase in the next years to reach 152 million by 2050 [1]. The pathogenesis of AD mainly relies on the presence of two main types of lesions in the brain: amyloid plaques (or senile plaques) and neurofibrillary tangles. However, the mechanisms leading to these disorders and their chronological apparition are still poorly understood and solved. These two lesions concomitantly appear in association with mitochondrial and vascular dysfunction, neuroinflammation, oxidative stress that eventually leads to neurodegeneration, firstly appearing in cholinergic regions [2]. To treat the symptoms of $\mathrm{AD}$, current drugs on the market are mainly AChEIs, and also include NMDA receptor antagonists. If moderately active along the progression of the disease, these drugs mostly improve cognitive functions and overall health of patients diagnosed with $\mathrm{AD}$ [3].
\end{abstract}

\section{Analysis of the clinical trials \& their failure}

Progress in the understanding of $\mathrm{AD}$ has, until now, not led to real advances in the discovery of agents able to treat or prevent $\mathrm{AD}$ as no agents have been approved by the US FDA since 2003. A recent analysis of clinical trials has listed more than 130 agents evaluated for the treatment of $\mathrm{AD}$ [4]. Interestingly the number of agents in each phase has increased compared with the 2018 pipeline. Most of the trials deal with the development of disease-modifying agents, however several trials also concern agents treating neuropsychiatric and behavioral symptoms or increasing cognitive functions. In all phases, amyloid pathology [5] remains the most common target with the development of novel small molecules, monoclonal antibodies or biological therapies. Such strategies have also been followed for anti-Tau agents in a lesser extent and for the development of 22 symptomatic agents or cognitive enhancers currently in phase III. We could also note the presence of several repurposing-drug [6] strategies as well innovative targets to limit multiple aging pathways, such as inflammation, vascular problems, or epigenetics. Despite all these efforts, $\mathrm{AD}$ drug development possesses one of the highest failure rates with no drug able to demonstrate drug-placebo difference. The lack of success in clinical trials could result from several causes, including problems with identifying the optimal drug for the right target, followed in its development with optimal biomarkers in a trial that includes relevant participant. In recent years some doubt has arisen concerning issues in the early development of several candidates. Among them, we could cite the use of inappropriate animal models in early development [7], some doubts about the validity of the initial targets, and also the complex stratification and diagnosis of the patients which should be in a state that allows them to benefit from an improvement in their health. Considering the multifactorial origin of $\mathrm{AD}$, the development of selective agents might not be sufficiently active to reverse the cognitive deficit. Indeed, the diversity of the pathogenetic elements at the origin of $\mathrm{AD}$ should be in favor of the development of combination therapy.

\section{Recent improvement to identify better candidates}

Facing these failures and taking into account the issues listed before, the FDA and authorities have recently set up a novel research framework to precise classification of patients, including predementia stages and mild 
cognitive impairment [8]. In order to improve this stratification, many trials are now based on the quantification of pathophysiological cerebrospinal fluid biomarkers $\left(A \beta_{1-42}, t-t a u, p-t a u_{18}\right.$, etc.) [9] or PET tracers developed for amyloid or tau identification [10]. The preclinical development of drugs should also evolve in order to take into account the complexity of $\mathrm{AD}$ in patients. As stated the animal models currently used, mainly transgenic mice, might not be really predictive of human disease. If other animal models are being developed, human organoids including stem-cells derived 3D tissue models of the brain would certainly be at the basis of the future preclinical testing to identify future valid targets and better candidates [11]. In order to tackle the complexity of AD, novel advances will surely focus on the identification of possible combination therapy [12]. This identification should be taken into account in the discovery process with novel screening strategies as well as in the future of the clinical evaluation. If the combination of drugs is a valuable strategy, the development of multiple ligands, also qualified as MultiTarget Directed Ligands is of great interest. Such compounds are single agent designed to modulate several biological targets of interest and numerous agents have been developed in recent years in an active field of research [13]. Many examples demonstrated potent activity in diverse animal models with promising safety results. Among them, several multiple agents have recently reached clinical trials and the recent conditional approval of oligomannate (GV-971) by the Chinese government's National Medical Product Administration might be the first example to be approved [14]. According to the putative mode of action of GV-971, the impact of the modulation of gut microbiota and neuroinflammation on AD is currently particularly studied [15]. Several novel therapeutic strategies and novel targets, including non-amyloid or non-tau strategy, should also be explored in order to develop disease-modifying or symptomatic agents $[16,17]$.

\section{Toward novel treatment for AD?}

The recent surprising announcement from Biogen and Eisai regarding the positive results of aducanumab [18] as a potential new treatment for $\mathrm{AD}$ represents a great achievement. Indeed, for the first time a compound, developed with the use of amyloid PET imaging, is able to limit the amyloid pathology and more importantly shows efficacy to limit dementia scores of the patients treated in the Phase III of the clinical trials. If novel data and probably another Phase III will be necessary before any regulatory submission, aducanumab might be the first disease-modifying agent able to reach this stage validating for the first time the antiamyloid strategy. This first step toward the approval of novel treatment for $\mathrm{AD}$ will also influence the development of other treatment currently under evaluation including other amyloid-targeted antibody such as BAN2401 also developed by Eisai and Biogen [19]. However if promising, it is unlikely that these agents will alone be able to limit or cure this complex pathology. We could postulate that the future of $\mathrm{AD}$ drug discovery will include combination of drugs addressing multiple target pathways to tackle the various form of $\mathrm{AD}$ with precision medicine.

\section{Financial \& competing interests disclosure}

The authors have no relevant affiliations or financial involvement with any organization or entity with a financial interest in or financial conflict with the subject matter or materials discussed in the manuscript. This includes employment, consultancies, honoraria, stock ownership or options, expert testimony, grants or patents received or pending, or royalties.

No writing assistance was utilized in the production of this manuscript.

\section{References}

1. Patterson C. World Alzheimer report 2018. ADI, London 1-48 (2018). www.alz.co.uk/research/WorldAlzheimerReport2018.pdf

2. Lee WH, Loo CY, Bebawy M, Luk F, Mason RS, Rohanizadeh R. Curcumin and its derivatives: their application in neuropharmacology and neuroscience in the 21st century. Curr. Neuropharmacol. 11(4), 338-378 (2013).

3. Zemek F, Drtinova L, Nepovimova E et al. Outcomes of Alzheimer's disease therapy with acetylcholinesterase inhibitors and memantine. Expert Opin. Drug Saf. 13(6), 759-774 (2014).

4. Cummings JL, Lee G, Ritter A, Sabbagh M, Zhong K. Alzheimer's disease drug development pipeline: 2019. Alzheimers Dement. 5, 272-293 (2019).

5. Karran E, Mercken M, De Strooper B. The amyloid cascade hypothesis for Alzheimer's disease: an appraisal for the development of therapeutics. Nat. Rev. Drug Discov. 10(9), 698-712 (2011).

6. Appleby BS, Cummings JL. Discovering new treatments for Alzheimer's disease by repurposing approved medications. Curr. Top. Med. Chem. 13(18), 2306-2327 (2013). 
7. Li C, Briner A, Götz J. The search for improved animal models of Alzheimer's disease and novel strategies for therapeutic intervention. Future Med. Chem. 11(15), 1853-1857 (2019).

8. Cummings J. The National Institute on Aging - Alzheimer's Association framework on Alzheimer's disease: application to clinical trials. Alzheimers Dement. 15(1), 172-178 (2018).

9. Toschi N, Lista S, Baldacci F et al. Biomarker-guided clustering of Alzheimer's disease clinical syndromes. Neurobiol. Aging 83, 42-53 (2019).

10. Pontecorvo MJ, Devous MD Sr, Navitsky M et al. Relationships between flortaucipir PET tau binding and amyloid burden, clinical diagnosis, age and cognition. Brain 140(3), 748-763 (2017).

11. King A. The search for better animal models of Alzheimer's disease. Nature 559(7715), S13-S15 (2018).

12. Fessel J. Alzheimer's disease combination treatment. Neurobiol. Aging 63, 165 (2018)

13. Lalut J, Rochais C, Dallemagne P. Multiple ligands in neurodegenerative diseases Drug Selectivity 17, 477-508 (2017).

14. Wang X, Sun G, Feng T et al. Sodium oligomannate therapeutically remodels gut microbiota and suppresses gut bacterial amino acids-shaped neuroinflammation to inhibit Alzheimer's disease progression. Cell Res. 29(10), 787-803 (2019).

15. Panza F, Lozupone M, Solfrizzi V, Watling M, Imbimbo BP. Time to test antibacterial therapy in Alzheimer's disease. Brain 142(10), 2905-2929 (2019).

16. Khan A, Corbett A, Ballard C. Emerging treatments for Alzheimer's disease for non-amyloid and non-tau targets. Expert Rev. Neurother. 17(7), 683-695 (2017).

17. Lalut J, Karila D, Dallemagne P, Rochais C. Modulating 5-HT4 and 5-HT6 receptors in Alzheimer's disease treatment. Future Med. Chem. 9(8), 781-795 (2017).

18. Biogen. Biogen plans regulatory filing for aducanumab in Alzheimer's disease based on new analysis of larger dataset from Phase III studies (2019). http: //investors.biogen.com/news-releases/news-release-details/biogen-plans-regulatory-filing-aducanumab-alzheimers-disease

19. NIH US National Library of Medicine A study to confirm safety and efficacy of BAN2401 in participants with early Alzheimer's disease (clarity AD) (2019). https://clinicaltrials.gov/ct2/show/NCT03887455 
